\title{
Reticulocytes in untreated Obstructive Sleep Apnoea
}

\author{
O. Marrone1, A. Salvaggio1, M. Gioia², A. Bonanno1, M. Profita1, \\ L. Riccobono', A. Zito3, G. Insalaco', M.R. Bonsignore ${ }^{3}$
}

\begin{abstract}
Reticulocytes in untreated Obstructive Sleep Apnoea. O. Marrone, A. Salvaggio, M. Gioia, A. Bonanno, M. Profita, L. Riccobono, A. Zito, G. Insalaco, M.R. Bonsignore. Background and Aim. The short, repetitive hypoxaemic episodes observed in obstructive sleep apnoea (OSA) may determine small augmentations in mature red blood cells. It is unknown whether they affect reticulocyte release. This study explored whether the number and degree of maturation of circulating reticulocytes may be altered in OSA, possibly through the effect of erythropoietin.

Methods. Fifty male adult patients with suspected OSA, normoxic during wakefulness, were studied. After nocturnal polysomnography, a blood sample was withdrawn for blood cells count, erythropoietin, iron and transferrin determination. Reticulocyte concentration and degree of immaturity [high (H), medium $(\mathrm{M})$, or low (L)] were also determined. Immature reticulocyte fraction (IRF) was calculated as $(\mathbf{M}+\mathbf{H})$ percentage of reticulocytes.
\end{abstract}

Results. A wide range of OSA severity was found [apnoea/hypopnoea index (AHI): 44.3 \pm 30.4 , range 0.3-105; sleep time spent at oxyhaemoglobin saturation $<90 \%$ : $18.1 \pm 22.2 \%$, range $0-81 \%$ ]. Both reticulocyte count and IRF slightly exceeded the normal range. Patients with a reticulocyte concentration $>2 \%$ had higher EPO levels $(p<0.05)$, but not worse nocturnal desaturations, than those with values $<2 \%$. By contrast, subjects with IRF $<15 \%$ showed worse desaturations $(p<0.05)$, but similar EPO concentrations, when compared to subjects whose IRF was $<10 \%$. At univariate analysis, reticulocyte count correlated to erythropoietin, while IRF to transferrin saturation, BMI and OSA severity. At multiple regression, only lowest nocturnal oxygen saturation remained a significant contributor to IRF ( $\left.\mathrm{r}^{2} \mathbf{0 . 2 2 3}, p<0.05\right)$.

Conclusions. This data suggests that hypoxaemia due to OSA could influence the release of immature reticulocytes, but this effect is not mediated by erythropoietin. Monaldi Arch Chest Dis 2008; 69: 3, 107-113.

Keywords: Haematopoiesis, Nocturnal hypoxemia, Obstructive sleep apnoea, Erythropoietin.

1 National Research Council, Institute of Biomedicine and Molecular Immunology (IBIM), Palermo;

2 V. Cervello Hospital, Laboratory of Clinical Pathology, Palermo;

3 University of Palermo, Department of Medicine, Pneumology, Physiology, and Nutrition (DIMPEFINU), Palermo, Italy.

This study was supported by the Italian National Research Council, order number ME.P01.006.

Correspondence: Dr. Oreste Marrone, Consiglio Nazionale delle Ricerche, Istituto di Biomedicina e Immunologia Molecolare, Via Ugo La Malfa 153, 90146 Palermo, Italy; e-mail: marrone@ibim.cnr.it

\section{Introduction}

One of the consequences of hypoxaemia is an increased erythropoietin (EPO) production, activated by the hypoxia-inducible factor-1 (HIF-1) [1]. EPO, in turn, acts as an antiapoptotic factor for erythroid progenitor cells and increases red blood cells (RBC) production [2]. At the same time, EPO increases the percentage of reticulocytes in peripheral blood, and, possibly, their more immature forms [3-5]. Immature reticulocytes increase particularly when iron availability is insufficient in proportion to the erythropoietic activity $[4,6]$.

Hypoxaemia may also contribute to the release of immature reticulocytes. The finding of an increase in peripheral immature reticulocytes in normal subjects after strenuous exercise [7] suggested a contribution of acute hypoxaemia to stimulate their release, independent of any EPO augmentation. In addition, an increase in immature reticulocytes was found in large samples of patients with pulmonary disease, who, however, had not been characterised for severity of hypoxaemia [8]. In patients with hypoxaemic respiratory disease, EPO and $\mathrm{RBC}$ responses to hypoxaemia appear highly variable, and apparently modulated by the type of disease and interindividual differences among patients with the same disease. For similar levels of hypoxaemia, patients with chronic obstructive pulmonary disease (COPD) produce more $\mathrm{RBC}$ than patients with idiopathic pulmonary fibrosis despite similar levels of EPO [9], but even among COPD patients intensity of erythropoiesis is highly variable [10] and erythropoietin resistance may occur [11].

Conversely from other respiratory disease characterised by persistent hypoxaemia, obstructive sleep apnoea (OSA) is characterised by repeated short hypoxaemic episodes during sleep. Studies performed in OSA have not clarified whether recurrence of hypoxaemia may affect erythropoiesis. In uncomplicated OSA polycythemia is rare. A relationship between nocturnal hypoxaemia and haematocrit may be demonstrated only on very large samples of OSA patients and its clin- 
ical significance is uncertain [12, 13]. Furthermore, treatment of OSA with continuous positive pressure ventilation (CPAP) prevented the acute increase in morning haematocrit secondary to nocturnal haemoconcentration, but did not otherwise affect it in the long term [14]. As regards EPO, only one group found that it was significantly related to OSA [15] and decreased after CPAP therapy [16]; other studies found that EPO was not significantly [17-20], or was only weakly associated to OSA [21]; more recently, a short-lived increase in EPO concentration after exposure to apnoeas has been shown [22], probably due to the short halflife of EPO. In two studies, EPO levels in OSA did not significantly correlate to $\operatorname{RBC}[16,20]$.

Investigations performed so far have not included the assessment of reticulocyte count and maturity in peripheral blood, which could better clarify the effects of OSA on erythropoietic activity. Automatic detection of immature reticulocytes can be easily obtained by evaluation of cellular fluorescence, which reflects RNA content: highly immature reticulocytes are characterised by high fluorescence.

The aim of this study was to explore whether OSA could affect erythropoiesis in patients with suspected OSA. Reticulocyte assessment, never included in previous studies on this topic, was obtained by measuring peripheral reticulocyte counts and maturity. The latter were correlated to severity of nocturnal respiratory disorders, morning plasma levels of EPO and iron availability as evaluated by transferrin saturation.

\section{Patients and methods}

Consecutive male subjects referred to the sleep laboratory of our Institution for nocturnal monitoring due to suspected OSA were studied. Exclusion criteria were pathological haemoglobins, anaemia ( $\mathrm{Hb}<13 \mathrm{~g} \%$ ), regular blood donations, wake oxyhaemoglobin saturation $\left(\mathrm{SaO}_{2}\right)<92 \%$, airway obstruction at spirometry $\left(\mathrm{FEV}_{1} / \mathrm{FVC}<70 \%\right)$. Fifty subjects were asked to participate, and all of them accepted. Among them, forty-seven did not regularly assume any medication, three assumed omeprazole, one simvastatin and one aspirin (100 $\mathrm{mg} /$ day). Fifteen patients were current smokers: among them, seven smoked $\leq 10$, four 11 to 20 , and four $>20$ cigarettes/day.

The protocol was approved by the local Ethical Committee and all patients gave written informed consent.

Patients underwent all-night monitoring for OSA diagnosis. 24 received home monitoring using a validated portable cardio-respiratory monitor (POLYMESAM, MAP, Martinsried, Germany), while 26 were recorded by laboratory polysomnography (PS-2 Compumedics, Abbottsford, Australia). The following morning, within two hours after the end of the nocturnal recording, in the fasting state, a venous blood sample was withdrawn.

Nocturnal studies were manually scored. In the polysomnographic studies, sleep was scored according to standard rules [23]. In the home record- ings, wakefulness periods were identified based on patients' diary and on characteristics of the recorded signals (like movement artefacts, heart rate behaviour, posture) and were excluded from the analysis. In all recordings, flow was detected by nasal cannulas connected to a pressure transducer. Apnoeas were scored when the airflow signal disappeared for at least 10 seconds, and were classified as central, obstructive or mixed, according to usual criteria. Hypopnoeas were considered as discernible reductions in the amplitude of the airflow signal for at least ten seconds, followed by a $>3 \%$ desaturation. Apnoea/hypopnoea index (AHI) was calculated as number of (apnoeas + hypopnoeas)/hour of sleep time, either measured on EEG (for polysomnographic registrations), or estimated (for cardiorespiratory monitorings). $\mathrm{SaO}_{2}$ was evaluated as baseline wake $\mathrm{SaO}_{2}$ value, absolute lowest nocturnal $\mathrm{SaO}_{2}$ (lowest $\mathrm{SaO}_{2}$ ), and sleep time with $\mathrm{SaO}_{2}<90 \%$ (TST<90\%). Patients were classified as non-OSA (AHI <5), or affected by mild, moderate or severe OSA according to an AHI 5-15, >15-30, or $>30$, respectively.

Venous blood in EDTA was used to determine red blood cell counts, haematocrit, haemoglobin, and reticulocytes. Reticulocytes were automatically counted (ADVIA 120, Bayer Diagnostics), based on the measurement of scatter and absorption of laser light. RNA content was analysed by the oxazine 750 method [24]. Fractions of reticulocytes with low (LRF), medium (MRF) and high (HRF) fluorescence for RNA (representing, respectively, subpopulations with a low, medium and high degree of immaturity) were determined. Immature reticulocyte fraction (IRF) was calculated as MRF + HRF. Iron and transferrin were measured, and transferrin saturation was calculated. EPO was measured by Elisa (R\&D Systems, Minneapolis, MN, USA).

Data is shown as mean $\pm \mathrm{SD}$. Linear regression analysis was applied to correlate the studied variables. Factorial one-way ANOVA followed by Student-Newman-Keuls test were used for comparisons between groups showing different \% of reticulocytes or IRF. Multiple regression was used to identify significant contributors to IRF, after exclusion of variables with high collinearity $(r>0.5)$. A $p<0.05$ was considered significant in all analyses.

\section{Results}

The patients studied were middle-aged $(46.1 \pm 9.0$ yrs), overweight or obese (BMI: $32.7 \pm 5.4 \mathrm{~kg} / \mathrm{m}^{2}$ ), and showed normal wake $\mathrm{SaO}_{2}(95.8 \pm 1.1 \%)$ and pulmonary function tests $\left(\mathrm{FEV}_{1} \%\right.$ predicted: $\left.104.9 \pm 11.9, \mathrm{FEV}_{1} / \mathrm{FVC} 80.5 \pm 4.5 \%\right)$. The range of severity of sleep respiratory disorders was quite wide, as shown in table 1 . According to AHI, 5 subjects were classified as non-OSA, 8 as mild OSA, 5 as moderate OSA, and 35 as severe OSA. REM sleep was recorded in all the 26 subjects who underwent polysomnography and accounted for $13.3 \pm 6.1 \%$ of total sleep time.

Data relevant to red blood cells and plasma determinations is shown in table 2. Pearson's corre- 
lation coefficients for haematological parameters are shown in table 3. Several significant relationships were found, but all correlations were weak. RBC counts correlated to EPO and TST $<90 \%$, while haematocrit showed a borderline correlation with EPO ( $p=.055)$. Reticulocytes, both as number of elements/litre and as \%RBC, were significantly correlated to EPO, but not to polysomnographic parameters, transferrin saturation or anthropometric characteristics. After patients were divided into three groups according to reticulocyte concentration (group 1: <1.5\%; group 2: $1.5-2 \%$; group 3: $>2 \%$ ) significant differences between groups were observed in EPO, but not in severity of oxygen desaturation (figure 1). By contrast, IRF did not correlate to EPO, while correlated significantly to all the considered parameters of severity of sleep respiratory disorders, transferrin saturation, and BMI. Among reticulocyte fractions, MRF was the most tightly correlated to the previous variables. HRF did not correlate to any variable. If patients were divided into three groups according to IRF (group 1: <10\%; group 2: 10-15\%; group 3: >15\%) significant differences between groups were observed in severity of oxygen desaturation, but not in EPO (figure 2).

Table 1. - Sleep Data

\begin{tabular}{lccc}
\hline & mean & SD & range \\
\hline TST (min) & 391 & 57.4 & $228-499$ \\
$\mathrm{AHI}(\mathrm{no} / \mathrm{h})$ & 44.3 & 30.4 & $0.3-105$ \\
$\mathrm{TST}<90 \%(\%)$ & 18.1 & 22.2 & $0-81$ \\
Lowest $\mathrm{SaO}_{2}(\%)$ & 75.0 & 13.5 & $44-92$ \\
\hline
\end{tabular}

TST: total sleep time; AHI: apnoea/hypopnoea index; TST<90\%: percentage of total sleep time spent with oxyhaemoglobin saturation below $90 \%$; Lowest $\mathrm{SaO}_{2}$ : lowest nocturnal oxyhaemoglobin saturation.

Table 2. - Haematological Data

\begin{tabular}{lccc}
\hline & mean & SD & range \\
\hline RBC $\left(\mathrm{n}^{*} 106 / \mathrm{ml}\right)$ & 5.3 & 0.3 & $4.7-6.1$ \\
$\mathrm{Hb}(\mathrm{g} \%)$ & 15.7 & 0.8 & $14.2-17.7$ \\
$\mathrm{Ht}(\%)$ & 45.5 & 2.4 & $41.1-51.9$ \\
Reticulocytes $(\mathrm{n} * 109 / 1)$ & 84.9 & 20.1 & $47.2-132.5$ \\
Reticulocytes $(\% \mathrm{RBC})$ & 1.6 & 0.4 & $0.9-2.7$ \\
LRF (\%) & 87.8 & 4.5 & $78.0-94.8$ \\
$\mathrm{MRF}(\%)$ & 10.8 & 3.9 & $4.1-19.5$ \\
$\mathrm{HRF}(\%)$ & 1.4 & 1.0 & $0.0-5.6$ \\
IRF (\%) & 12.3 & 4.5 & $5.2-22.0$ \\
Transferrin saturation $(\%)$ & 23.8 & 7.3 & $12.7-43.0$ \\
EPO (mIU/ml) & 10.9 & 3.8 & $5.1-21.7$ \\
\hline
\end{tabular}

RBC: red blood cells; Hb: haemoglobin; Ht: haematocrit; LRF: low immaturity reticulocyte fraction; MRF: medium immaturity reticulocyte fraction; HRF: high immaturity reticulocyte fraction; IRF: immature reticulocyte fraction; EPO: erythropoietin; IRF: immature reticulocyte fraction.

Table 3. - Univariate correlations

\begin{tabular}{lcccccc}
\hline & AHI & Lowest $\mathbf{S a O}_{\mathbf{2}}$ & TST<90\% & EPO & Transf sat & BMI \\
\hline RBC & .278 & -.195 & $.280^{*}$ & $-.282^{*}$ & .032 & .232 \\
Ht & .154 & -.115 & .195 & -.273 & .241 & .192 \\
Ret & .027 & -.160 & .240 & $.287^{*}$ & -.153 & -.019 \\
Ret\% & -.027 & -.133 & .175 & $.363^{* *}$ & -.171 & -.060 \\
IRF & $.327^{*}$ & $-.375^{* *}$ & $.326^{*}$ & .134 & $-.339^{*}$ & $.323^{*}$ \\
LRF & $-.327^{*}$ & $.374^{* *}$ & $-.325^{*}$ & -.133 & $.338^{*}$ & $-.322^{*}$ \\
MRF & $.388^{* *}$ & $-.445^{* *}$ & $.390^{* *}$ & .132 & $-.391^{* *}$ & $.387^{* *}$ \\
HRF & -.020 & .021 & -.032 & .096 & -.040 & -.037 \\
\hline
\end{tabular}

AHI: apnoea/hypopnoea index; Lowest $\mathrm{SaO}_{2}$ : lowest nocturnal oxyhaemoglobin saturation; TST<90\%: percentage of total sleep time spent with oxyhaemoglobin saturation below 90\%; EPO: erythropoietin; Transf sat: transferrin saturation; BMI: body mass index; RBC: red blood cells; Ht: haematocrit; Ret: reticulocyte concentration; Ret\%: reticulocytes as \% of RBC; IRF: immature reticulocyte fraction; LRF: low immaturity reticulocyte fraction; MRF: medium immaturity reticulocyte fraction; HRF: high immaturity reticulocyte fraction.

$* p<.05 . * * p<.01$ 


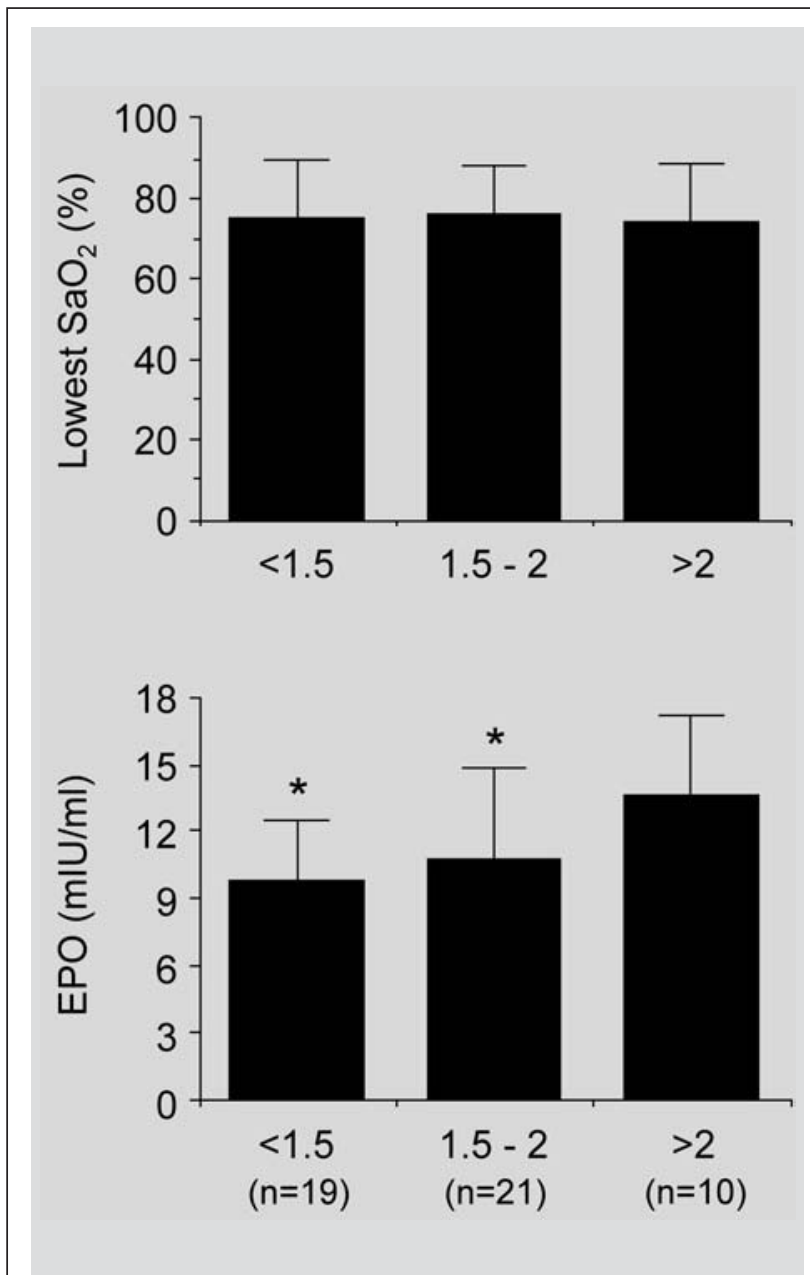

Fig. 1. - Lowest nocturnal oxyhemoglobin saturation (upper panel) and morning erythropoietin concentration (lower panel) in groups of patients with different $\%$ of reticulocytes. $* p<0.05$ vs group 3.

AHI, TST $<90 \%$, and lowest $\mathrm{SaO}_{2}$ were highly intercorrelated $(r>.5$ for all). Therefore, only lowest $\mathrm{SaO}_{2}$, which showed the highest correlation coefficient with IRF, together with BMI and transferrin saturation was entered as independent variable at multiple regression with IRF as dependent variable. Only lowest $\mathrm{SaO}_{2}$ proved a significant contributor to IRF at that test $\left(\mathrm{r}^{2}=.223\right)$.

Characteristics of sleep respiratory disorders and haematological data according to smoking habits are shown in table 4. Among subjects smoking >10 cigarettes/day, two were non-OSA and six showed severe OSA. If these eight subjects were excluded from analyses, only the correlation between IRF and transferrin saturation was lost, while all the others remained significant.

\section{Discussion}

To our knowledge, this is the first study that evaluated reticulocytes in OSA. The studied sample included subjects with a wide spectrum of sleep respiratory disorders severity, ranging from normal to very severe. The data suggests a minor effect of OSA on eryhtropoiesis, in line with previous studies $[12,13]$. In particular, intermittent hypoxaemia may affect the release of immature reticulocytes,

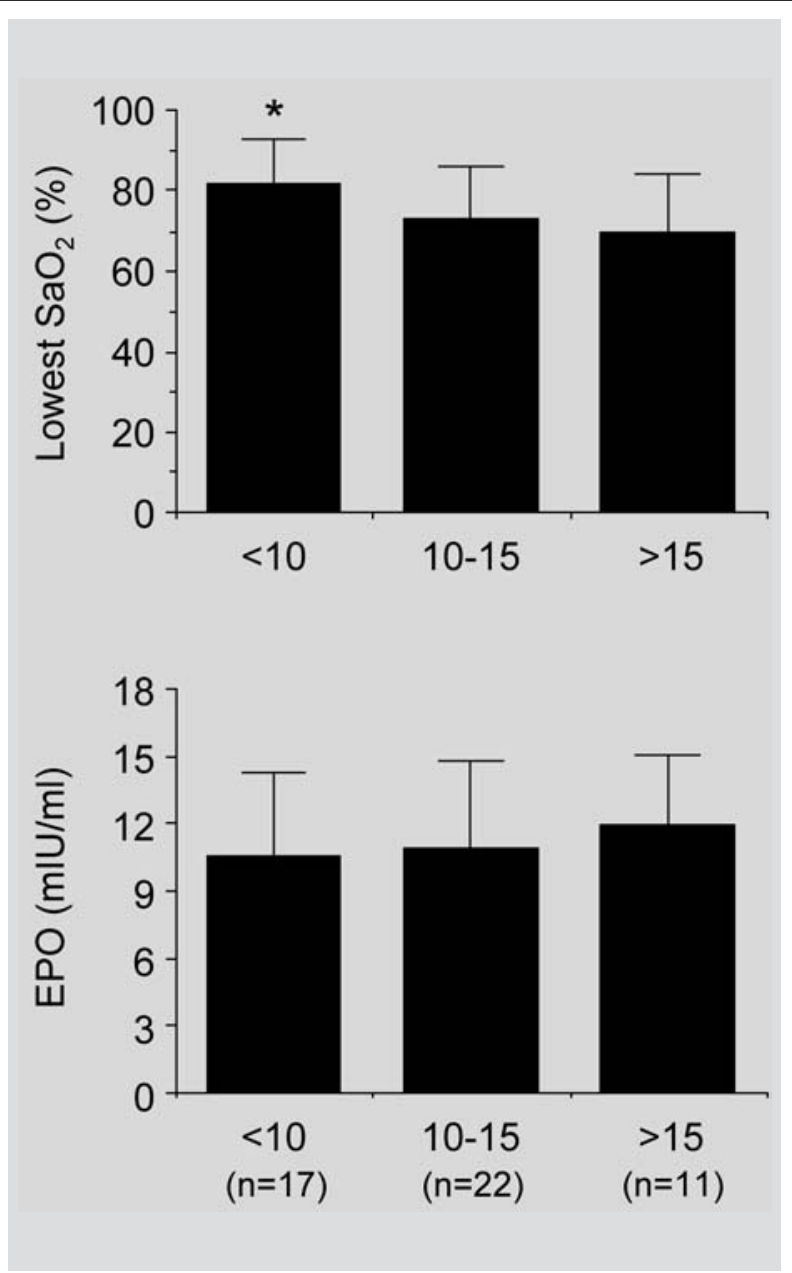

Fig. 2. - Lowest nocturnal oxyhemoglobin saturation (upper panel) and morning erythropoietin concentration (lower panel) in groups of patients with different immature reticulocyte fractions (IRF). ${ }^{*} p<0.05$ vs group 3 .

possibly by a direct effect on the bone marrow rather than through the action of EPO.

Although the findings of this study indicate some influence of OSA on peripheral reticulocytes release, there is much uncertainty on the normal range of reticulocyte values, as they may change in relationship to several factors, like age, gender or equipment used for the determinations. We are aware of only one study using the ADVIA system for reticulocyte determinations with results separately reported for normal adult male subjects [25]: such results showed lower reticulocyte number and immaturity than in the subjects of the present study (normal values reported: reticulocyte number: 29-69 x103/L; reticulocyte \%RBC: 0.5-1.4; MRF: $1.5-10.7 \%$; HRF: 0-2.0\%). Other results of reticulocytes determinations were more similar to those found in this study, but they were in relation to samples including children and ill subjects [26, 27 , or were obtained with instruments other than the ADVIA system [28].

A weak correlation between degree of reticulocyte maturity and severity of nocturnal hypoxaemia, independent of iron availability, was found. The number of reticulocytes, but not their degree of maturity, correlated to EPO concentration, which, in turn, did not correlate to OSA severity. 
Table 4. - Sleep respiratory disorders and haematological data according to smoking habits

\begin{tabular}{|c|c|c|c|c|c|c|}
\hline & \multicolumn{2}{|c|}{$\begin{array}{c}\text { non-smokers } \\
\mathbf{n}=35\end{array}$} & \multicolumn{2}{|c|}{$\begin{array}{c}\text { smokers }<10 \text { cig./day } \\
n=7\end{array}$} & \multicolumn{2}{|c|}{$\begin{array}{c}\text { smokers }>10 \text { cig./day } \\
n=8\end{array}$} \\
\hline & mean & SD & mean & SD & mean & SD \\
\hline AHI (n/h) & 41.3 & 30.4 & 44.2 & 20.4 & 58.4 & 36.9 \\
\hline Lowest $\mathrm{SaO}_{2}(\%)$ & 75.3 & 13.1 & 78.1 & 14.2 & 72.1 & 15.8 \\
\hline TST $<90 \%(\%)$ & 16.7 & 22.7 & 16.4 & 26.6 & 29.1 & 22.0 \\
\hline $\mathrm{RBC}\left(10^{6} / \mathrm{ml}\right)$ & 5.3 & 0.3 & 5.4 & 0.3 & 5.2 & 0.3 \\
\hline $\mathrm{Hb}(\mathrm{g} \%)$ & 15.6 & 0.9 & 16.0 & 0.9 & 15.6 & 0.9 \\
\hline $\mathrm{Ht}(\%)$ & 45.4 & 2.5 & 46.1 & 2.7 & 45.1 & 2.1 \\
\hline Ret $\left(10^{9} / 1\right)$ & 85.6 & 21.1 & 77.7 & 19.9 & 88.2 & 15.7 \\
\hline $\operatorname{Ret}(\% \mathrm{RBC})$ & 1.6 & 0.4 & 1.5 & 0.4 & 1.7 & 0.3 \\
\hline $\operatorname{IRF}(\%)$ & 12.6 & 4.4 & 9.1 & 3.2 & 14.3 & 4.5 \\
\hline $\mathrm{EPO}(\mathrm{mIU} / \mathrm{ml})$ & 10.6 & 3.4 & 10.2 & 4.5 & 13.0 & 4.4 \\
\hline
\end{tabular}

Cig.: cigarettes; AHI: apnoea/hypopnoea index; Lowest $\mathrm{SaO}_{2}$ : lowest nocturnal oxyhaemoglobin saturation; TST<90\%: percentage of total sleep time spent with oxyhaemoglobin saturation below 90\%; RBC: red blood cells; Hb: haemoglobin; Ht: haematocrit; Ret: reticulocytes; IRF: immature reticulocyte fraction; EPO: erythropoietin.

The possible effect of smoking was considered, and it did not appear to interfere with the results, in line with previous studies that did not demonstrate an increase in reticulocytes in smokers $[29,30]$.

The relationship between hypoxaemia and maturity of circulating reticulocytes has not been studied. Some clues about its possible influence may be obtained from a few studies on reticulocytes in conditions possibly associated with hypoxaemia, but where hypoxaemia was not measured. In particular, in patients with unspecified pulmonary disease reticulocytes percentage was not augmented while an increase in immature reticulocytes was reported; that was considered a possible effect of hypoxia and EPO, which, however, were not measured [8]. Besides, in normal healthy trained subjects, strenuous exercise was followed by an increase in both MRF and HRF, associated with decreased plasma EPO concentration [7]. A possible explanation for this finding is that exercise itself contributed to the increased immature reticulocyte release. However exercise could have acutely reduced oxygen availability, and, by this mechanism, could have increased circulating immature reticulocytes, although plasma EPO concentration fell. By analogy, in the OSA patients obstructed respiratory efforts could have played a role. However, the significant relationships between IRF and nocturnal hypoxaemia, that was independent of EPO, suggest that hypoxaemia directly exerted some effect on the release of immature reticulocytes. The changes were small and unlikely to represent a clinically significant effect. Similar to the influence of OSA severity on haematocrit [12], an effect on immature reticulocytes release in the peripheral blood could be clearly demonstrated only in a very large patients' sample. In this study, the presence of patients with very long periods of the night spent with $\mathrm{SaO}_{2}$ $<90 \%$ (up to $81 \%$ ) raises the question if stable rather than intermittent hypoxaemia could be required to find an effect on immature reticulocyte release. However, if we excluded from the analysis the seven patients with a TST $<90 \%$ greater than $50 \%$, the correlation between lowest $\mathrm{SaO}_{2}$ and IRF remained significant.

The lack of any relationship between the intensity or duration of nocturnal hypoxaemia and EPO deserves comment. Despite some conflicting results, previous literature has also shown a poor, if any, effect of OSA on plasma EPO [15, 17-20, 22]. This could, however, have different explanations.

One hypothesis could be that the pattern of intermittent hypoxaemia that characterises OSA is poorly effective in stimulating the release of this hormone, in line with the finding in vitro that intermittent hypoxia may preferentially activate a proinflammatory pathway instead than HIF-1 $\alpha$ pathway [31]. Most of our knowledge about the relationship between hypoxaemia and EPO has been obtained through studies on prolonged hypoxaemia. Experimental studies on intermittent hypoxaemia in humans, mainly performed in athletes, evaluated the effects of hypoxic exposure for a few hours a day, and showed conflicting results not only as regards EPO but also reticulocytes release: a few investigations showed both an increase in reticulocytes and in EPO [32-34], other studies found an increase in reticulocytes without a concomitant increase in EPO [35-36], and another group reported an increase in EPO without a concomitant increase in reticulocytes [37-39]. However, intermittent hypoxaemia of OSA consists of sequences of short hypoxic episodes, usually lasting less than a minute, separated by even shorter normoxic intervals, recurring up to hundreds of times each night during sleep. Only one study evaluated EPO response in normal subjects exposed to an intermittent hypoxaemia pattern resembling the one of OSA, and found an EPO response only for prolonged exposures to intermittent hypoxaemia [40]. According to this investigation, an EPO response should have been observed 
in the most severe cases, but that could not be demonstrated in the present study.

Alternatively, the one- to two-hours interval between morning awakening and blood withdrawal could have blunted any effect of nocturnal respiratory disorders on EPO. Actually, some studies suggested that some effect of OSA on EPO may be found, provided that blood is sampled shortly after a prolonged period of exposure to apnoeas [22]. However, as 'half-life' of EPO is about 4 hours, we believe this hypothesis to be unlikely. Besides, inflammatory cytokines, which are produced in excess in OSA $[41,42]$, could confound the relationship between EPO and hypoxaemia, as they have complex interactions with HIF and EPO, and may decrease their production or inhibit their activity $[43,44]$. Such hypothesis was tested by measuring TNF-alpha concentration in serum samples, but no correlation was found between TNF-alpha and EPO, reticulocyte number or maturity (data not shown).

EPO was significantly, although weakly, correlated to total RBC and reticulocyte counts. In fact, in two previous studies on OSA with only few subjects, no significant correlation between EPO and $\mathrm{RBC}$ was found $[16,20]$. The hypothesis that the effects of EPO might be inhibited by inflammatory cytokines is not supported by our findings.

In conclusion, OSA appears associated with a minor disturbance in erythropoietic activity. It could subclinically affect erythropoiesis by inducing a small increase in circulating immature reticulocytes, which is correlated to sleep hypoxaemia. Studies on larger patients' samples, as well as investigations on the effects of treatment of OSA, could better clarify the role of intermittent hypoxaemia on immature reticulocyte release.

Acknowledgement: The authors wish to thank Mr. Giovanni Sciortino for his technical support.

\section{References}

1. Huang LE, Bunn HF. Hypoxia-inducible factor and its biomedical relevance. J Biol Chem 2003; 278: 1957519578.

2. Fisher JW. Erythropoietin: physiology and pharmacology update. Exp Biol Med 2003; 118: 1-14.

3. Han YS, Kishimoto T. Reticulocyte maturity index reflects erythropoietin effects in hemodialysis patients. Osaka City Med J 1997; 43: 69-76.

4. Brugnara C. Reticulocyte cellular indices: a new approach in the diagnosis of anemias and monitoring of erythropoietic function. Crit Rev Clin Lab Sci 2000; 37: 93-130.

5. Al-Huniti NH, Widness JA, Schmidt RL, Veng Pedersen P. Pharmacodynamic analysis of changes in reticulocyte subtype distribution in phlebotomy-induced stress erythropoiesis. J Pharmacokinet Pharmacodyn 2005; 32: 359-376.

6. Choi JW, Pai SH. Reticulocyte subpopulations and reticulocyte maturity index (RMI) rise as body iron status falls. Am J Hematol 2001; 67: 130-135.

7. Morici G, Zangla D, Santoro A, et al. Supramaximal exercise mobilizes hematopoietic progenitors and reticulocytes in athletes. Am J Physiol Regul Integr Comp Physiol 2005; 289: R1496-R1503.
8. Kendall RG, Mellors I, Hardy J, Mardle B. Patients with pulmonary and cardiac disease show an elevated proportion of immature reticulocytes. Clin Lab Haematol 2001; 23: 27-31.

9. Tassiopoulos S, Kontos A, Kostantinopoulos K, et al. Erythropoietic response to hypoxaemia in diffuse pulmonary fibrosis, as opposed to chronic obstructive pulmonary disease. Respir Med 2001; 95: 471-475.

10. Similowski T, Agustì A, MacNee W, Schönhofer B. The potential impact of anaemia of chronic disease in COPD. Eur Respir J 2006; 27: 390-396.

11. John M, Hoernig S, Doehner W, Okonko DD, Witt C, Anker SD. Anemia and inflammation in COPD. Chest 2005; 127: 825-829.

12. Hoffstein V, Herridge M, Mateika S, Redline S, Strohl KP. Hematocrit levels in sleep apnea. Chest 1994; 106: 787-791.

13. Choi JB, Loredo JS, Norman D, et al. Does obstructive sleep apnea increase hematocrit? Sleep Breath 2006; 10: $155-160$.

14. Krieger J, Sforza E, Delanoe C, Petiau C. Decrease in haematocrit with continuous positive airway pressure treatment in obstructive sleep apnoea patients. Eur Respir J 1992; 5: 228-233.

15. Cahan C, Decker MJ, Arnold JL, et al. Diurnal variations in serum erythropoietin levels in healthy subjects and sleep apnea patients. J Appl Physiol 1992; 72: 2112-2117.

16. Cahan C, Decker MJ, Arnold JL, Goldwasser E, Strohl KP. Erythropoietin levels with treatment of obstructive sleep apnea. J Appl Physiol 1995; 79: 1278-1285.

17. McKeon JL, Saunders NA, Murree-Allen K, et al. Urinary uric acid:creatinine ratio, serum erythropoietin, and blood 2,3-diphosphoglycerate in patients with obstructive sleep apnea. Am Rev Respir Dis 1990; 142: 8-13.

18. Goldman JM, Ireland RM, Berthon-Jones M, Grunstein RR, Sullivan CE, Biggs JC. Erythropoietin concentrations in obstructive sleep apnoea. Thorax 1991; 46: 25-27.

19. Pokala P, Llanera M, Sherwood J, Scharf S, Steinberg H. Erythropoietin response in subjects with obstructive sleep apnea. Am J Respir Crit Care Med 1995; 151: 1862-1865.

20. Carlson JT, Hedner J, Fagerberg D, Ejnell H, Magnusson B, Fyhrquist F. Secondary polycythemia associated with nocturnal apnoea. A relationship not mediated by erythropoietin? J Intern Med 1992; 231: 381-387.

21. Imagawa S, Yamaguchi Y, Higuchi M, et al. Levels of vascular endothelial growth factor are elevated in patients with obstructive sleep apnea-hypopnea syndrome. Blood 2001; 98: 1255-1257.

22. Winniki M, Shamsuzzaman A, Lanfranchi P, et al. Erythropoietin and obstructive sleep apnea. Am J Hypertens 2004; 17: 783-786.

23. Rechtschaffen A, Kales A. A manual of standardized terminology, techniques, and scoring system for sleep stages of human subjects. Los Angeles, CA: BIS/BRI, UCLA, 1968.

24. D'Onofrio G, Chirillo R, Zini G, Caenaro G, Tommasi M, Micciulli G. Simultaneous measurement of reticulocyte and red blood cell indices in healthy subjects and patients with microcytic and macrocytic anemia. Blood 1995; 85: 818-823.

25. Van den Bossche J, Devreese K, Malfait R, et al. Reference intervals for a complete blood count determined on different automated haematology analysers: Abx Pentra 120 Retic, Coulter Gen-S, Sysmex SE 9500, Abbott Cell Dyn 4000 and Bayer Advia 120. Clin Chem Lab Med 2002; 40: 69-73.

26. Buttarello M, Bulian P, Farina G, et al. Flow cytometric reticulocyte counting. Parallel evaluation of five ful- 
ly automated analyzers: an NCCLS-ICSH approach. Am J Clin Pathol 2001; 116: 100-111.

27. Buttarello M, Bulian P, Farina G, Petris MG, Temporin $\mathrm{V}$, Toffolo L. Five fully automated methods for performing immature reticulocyte fraction. Comparison in diagnosis of bone marrow aplasia. Am J Clin Pathol 2002; 117: 871-879.

28. Banfi G, Mauri C, Morelli B, Di Gaetano N, Malgeri U, Melegati G. Reticulocyte count, mean reticulocyte volume, immature reticulocyte fraction, and mean sphered cell volume in elite athletes: reference values and comparison with the general population. Clin Chem Lab Med 2006; 44: 616-622.

29. Tarallo P, Humbert JC, Mahassen P, Fournier B, Henny J. Reticulocytes: biological variations and reference limits. Eur J Haematol 1994; 53: 11-15.

30. Mercelina-Roumans PE, Ubachs JM, van Wersch JW. The reticulocyte count and its subfractions in smoking and non-smoking pregnant women. Eur J Clin Chem Clin Biochem 1995; 33: 263-5.

31. Ryan S, Taylor CT, McNicholas WT. Selective activation of inflammatory pathways by intermittent hypoxia in obstructive sleep apnea syndrome. Circulation 2005; 112: 2660-7.

32. Savourey G, Garcia N, Besnard Y, Guinet A, Hanniquet AM, Bittel J. Pre-adaptation, adaptation and de-adaptation to high altitude in humans: cardio-ventilatory and haematological changes. Eur J Appl Physiol Occup Physiol 1996; 73: 529-535.

33. Rodriguez FA, Ventura JL, Casas M, et al. Erythropoietin acute reaction and haematological adaptations to short, intermittent hypobaric hypoxia. Eur J Appl Physiol 2000; 82: 170-177.

34. Villa JG, Lucia A, Marroyo JA, et al. Does intermittent hypoxia increase erythropoiesis in professional cyclists during a 3-week race? Can J Appl Physiol 2005; 30: 61-73.

35. Garcia N, Hopkins SR, Powell FL. Effects of intermit- tent hypoxia on the isocapnic hypoxic ventilatory response and erythropoiesis in humans. Respir Physiol 2000; 123: 39-49.

36. Savourey G, Launay JC, Besnard Y, et al. Control of erythropoiesis after high altitude acclimatization. Eur $J$ Appl Physiol 2004; 93: 47-56.

37. Ashenden MJ, Gore CJ, Dobson GP, et al. Simulated moderate altitude elevates serum erythropoietin but does not increase reticulocyte production in welltrained runners. Eur J Appl Physiol 2000; 81: 428-435.

38. Abellán R, Remacha AF, Ventura R, Sardà MP, Segura J, Rodríguez FA. Hematologic response to four weeks of intermittent hypobaric hypoxia in highly trained athletes. Haematologica 2005; 90: 126-127.

39. Gore CJ, Rodriguez FA, Truijens MJ, Townsend NE, Stray-Gundersen J, Levine BD. Increased serum erythropoietin but not red cell production after 4 wk of intermittent hypobaric hypoxia (4,000-5,500 m). $J$ Appl Physiol 2006; 101: 1386-1393.

40. Knaupp W, Khilnani S, Sherwood J, Scharf S, Steinberg $\mathrm{H}$. Erythropoietin response to acute normobaric hypoxia in humans. J Appl Physiol 1992; 73: 837-840.

41. Vgontzas AN, Papanicolaou DA, Bixler EO, Kales A, Tyson K, Chrousos GP. Elevation of plasma cytokines in disorders of excessive daytime sleepiness: role of sleep disturbance and obesity. J Clin Endocrinol Metab 1997, 82: 1313-1316.

42. Minoguchi K, Yokoe $\mathrm{T}$, Tazaki $\mathrm{T}$, et al. Increased carotid intima-media thickness and serum inflammatory markers in obstructive sleep apnea. Am J Respir Crit Care Med 2005; 172: 625-630.

43. Haddad JJ, Harb HL. Cytokines and the regulation of hypoxia-inducible factor (HIF)-1 alpha. Int Immunopharmacol 2005; 5: 461-483.

44. Ferrucci L, Guralnik JM, Woodman RC, et al. Proinflammatory state and circulating erythropoietin in persons with and without anemia. Am J Med 2005; 118, 1288.

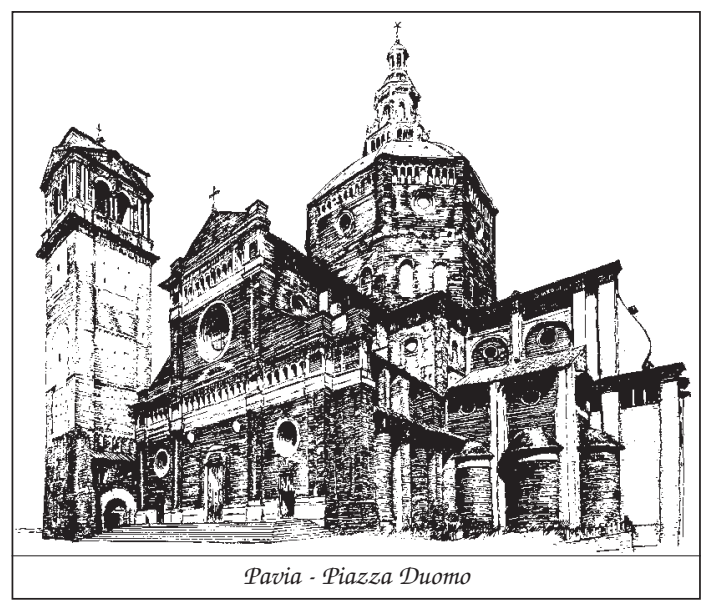

